\title{
Mathematical Platonism and the Nature of Infinity
}

\author{
Gilbert B. Côté \\ Sudbury, Ontario, Canada \\ Email: gbcote@gmail.com \\ Received April 25 $5^{\text {th }}, 2013$; revised May $25^{\text {th }}, 2013$; accepted June $2^{\text {nd }}, 2013$
}

Copyright (C) 2013 Gilbert B. Côté. This is an open access article distributed under the Creative Commons Attribution License, which permits unrestricted use, distribution, and reproduction in any medium, provided the original work is properly cited.

\begin{abstract}
An analysis of the counter-intuitive properties of infinity as understood differently in mathematics, classical physics and quantum physics allows the consideration of various paradoxes under a new light (e.g. Zeno's dichotomy, Torricelli's trumpet, and the weirdness of quantum physics). It provides strong support for the reality of abstractness and mathematical Platonism, and a plausible reason why there is something rather than nothing in the concrete universe. The conclusions are far reaching for science and philosophy.
\end{abstract}

Keywords: Mathematical Platonism; Infinity; Zeno; Torricelli; Abstractness; Quantum Physics; Dichotomy; Trumpet; Paradox

\section{Introduction}

The ontology of mathematical Platonism remains to this day an unresolved issue. Most philosophers and mathematicians concede that numbers exist and are abstract, but there is considerable divergence of opinion on whether they exist independently of rational observers (Cole, 2010). The difficulty is particularly evident when one considers the concept of numerical infinity and its counter-intuitive properties in the different fields of mathematics, classical physics and quantum physics.

In the text below, I will endeavour to tease apart the various meanings of infinity, in an attempt to clear the confusion that otherwise produces various contradictions and well known paradoxes. The proposed solutions should illuminate the philosophical debate on the reality of abstractness and the significance of mathematical Platonism.

\section{Infinity and Mathematics}

Mathematical infinity is clearly defined on the basis of the original and authoritative work of German mathematician Georg Cantor (1845-1918) (Dunham, 1990, 1994). The first type of mathematical infinity that comes to mind is that of the natural numbers (also called positive integers: 1, 2, 3, 4, etc.). They are infinite because we can always add 1 to any large integer previously identified. Any set of numbers that can be put in a one-to-one correspondence with the positive integers is also said to be denumerably (countably) infinite because we can identify all its members one at a time. This turns out to be the case for the odd integers, the even integers, for the set of negative and positive integers and 0 , the rational numbers (those that are the ratio of two integers), the prime numbers (those that can only be divided by themselves and 1) and all algebraic numbers (the roots of non-zero polynomial equations).

All these sets of numbers have the same infinite size or "cardinality", formally denoted as $\aleph_{0}$ (aleph-naught). This is very counter-intuitive because at first sight the number of all positive integers (odd and even) would appear to be twice as large as the number of even integers. However, infinite sets of numbers do not behave like finite sets. To compare the cardinality of the set of all integers with that of all even integers, we start with the first member of the set of all integers (i.e. 1) and the first member of the set of all even integers (i.e. 2) to establish the unique correspondence $1 \leftrightarrow 2$. We then continue sequentially with $2 \leftrightarrow 4$, $3 \leftrightarrow 6,4 \leftrightarrow 8,5 \leftrightarrow 10$, etc. and find that we can carry on indefinitely and establish a unique one-to-one correspondence between all members of the two sets. For each member of one set, there is one and only one corresponding member in the other set. This proof demonstrates that both sets have the same cardinality.

Several infinite sets of numbers have higher cardinality than those above because a one-to-one correspondence with the positive integers cannot be established. These sets are said to be non-denumerable (non-countable, or innumerable) and contain more members than the denumerable sets do. Their cardinality is denoted as c. These sets include the set of real numbers contained in the interval between any two numbers, the set of irrationals (like $\sqrt{2}$ ), the set of transcendental numbers (i.e. nonalgebraic numbers like $\pi$ and $e$ ), the set of complex numbers (numbers with a real part and an imaginary part), the number of points in a square, and the number of points in any $n$-dimensional space.

In set theory, a power set is defined as the set of all subsets of a set, including an empty set and the original set. The cardinality of power sets is higher than that of their original sets. Power sets of non-denumerable sets thus have cardinality higher than $\aleph_{0}$ and $\mathbf{c}$. If we take the power sets of power sets and repeat the operation again and again, we can build an infinity of infinite sets, each set having higher cardinality than the precedent. This never-ending process looks like mathematical infinity feeding upon itself to produce ever greater degrees of infinity. This is the archetype of self-referral and is inherent to infinity. 


\section{Infinity and Classical Physics}

If we try to apply the notion of mathematical infinity to concrete reality, we run into endless conceptual difficulties and paradoxes. The best examples are more than 2400 years old and were originally presented by the Greek philosopher Zeno of Elea. His dichotomy paradox, for example, presents the apparent difficulty of travelling from point $\mathrm{A}$ to point $\mathrm{B}$, due to the fact that one must first reach the midpoint $\mathrm{C}$ between $\mathrm{A}$ and $\mathrm{B}$. Once at point $\mathrm{C}$, one must then reach a new midpoint between point $\mathrm{C}$ and point $\mathrm{B}$. New midpoints thus appear ad infinitum, preventing the tired traveller from ever reaching point $\mathrm{B}$.

Two main solutions have been proposed along the ages. Practically, one can simply brush off the paradox by walking from A to B and beyond, apparently demonstrating that Zeno was wrong. The solution is pragmatic but ignores rather than answers Zeno's question about what happens when one travels an infinite number of small stretches along a Euclidian line.

A more sophisticated mathematical reply consists of using the infinite series

$$
\sum_{n=1}^{\infty}\left(\frac{1}{2}\right)^{n}=1
$$

to show that, at the limit, the sum of this infinite sequence of diminishing distances converges to the whole A-B distance. This is a counter-intuitive but solid demonstration that the sum of an infinite number of values can add up to a finite value. It is often claimed to solve Zeno's dichotomy paradox but on the contrary, it simply illustrates it by showing that the sum tends to the limit without ever reaching it. Strictly speaking, mathematical infinity has no end, so point B gets closer and closer along the series but is never reached (unless we truncate the process and abandon infinity).

The essential problem with the dichotomy paradox is that it follows Euclid instead of Democritus and Planck. The appropriate answer to Zeno is that in practice, we cannot divide our travel in an infinite number of diminishing halves because distances are not continuous. We now know that roads are not endlessly divisible, because the concrete world is made of indivisible atoms (or sub-atomic particles). We must not confuse Euclidian geometry with the physical world. An infinitely long Euclidian line can be divided ad infinitum, but a road or any piece of concrete matter cannot. Concrete distances can be very small but not infinitely so. In modern physics the smallest possible distance is known as Planck length and is equal to $1.616 \times$
$10^{-35}$ meters. Similarly, the shortest period of time is known as Planck time and is equal to $5.39 \times 10^{-44}$ seconds. If parts of space-time cannot be infinitely small, instants of time cannot either.

Mathematically speaking, anything that cannot be divided infinitely cannot be infinitely large. Accordingly, our concrete universe is definitely not infinitely large and will not last forever. It is astronomically large and amazingly old, but not infinitely so (especially if it started at the Big Bang). We do not have the capacity to count its galaxies but their number is not mathematically infinite. Our universe will last for eons, but not forever. In other words, mathematical infinity does not exist in our concrete, finite universe.

\section{Infinity and Torricelli's Trumpet}

Torricelli's trumpet is another renowned paradox dealing with infinity (Clegg, 2003; Weisstein, 2013). Its careful consideration will help us firmly establish the difference between the abstract realm of mathematics and the concrete world. The trumpet was first described by the Italian mathematician Evangelista Torricelli (1608-1647). First consider the graph of the equation $y=1 / x$ for all values of $x \geq 1.0$, as shown in Figure 1 .

Then rotate the graph around the $\mathrm{x}$ axis to obtain the trumpet shown in Figure 2. Note that the trumpet has a flared bell of diameter 2.0 and is infinitely long.

Torricelli's trumpet is also known as Gabriel's horn, in reference to the biblical Archangel Gabriel who is said to use a horn to announce God's news; it seems fitting to equip an archangel with an infinitely long horn. The paradox stems from the fact that the horn apparently has an infinite surface but a finite volume. Indeed, it has been clearly shown mathematically that as x approaches infinity, the horn's surface area also tends to infinity, while the volume enclosed by the curve tends towards the value of $\pi$ cubic units (where $\pi=3.1416 \cdots$ is conventionally considered to be a finite value between 3 and 4). The existence of a finite volume bound by an infinite area is the paradox that is still considered unresolved today.

Gabriel's horn cannot concretely exist. It is longer than the diameter of the universe, and no one can blow in it since it goes on endlessly without ever reaching a mouthpiece. In practice, we could not fill it up completely with a quantity $\pi$ of real paint, or cover its interior with an infinite amount of real paint since the paint molecules would be too large to squeeze into the infinitely thin extremity.

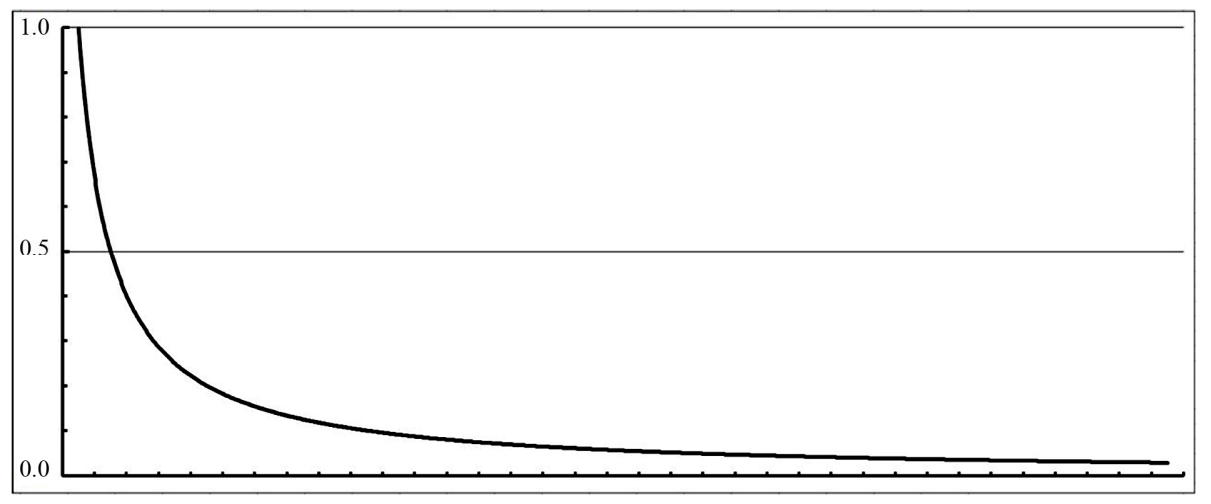

Figure 1.

Graphical representation of the equation $y=1 / x$ for $x \geq 1.0$. 


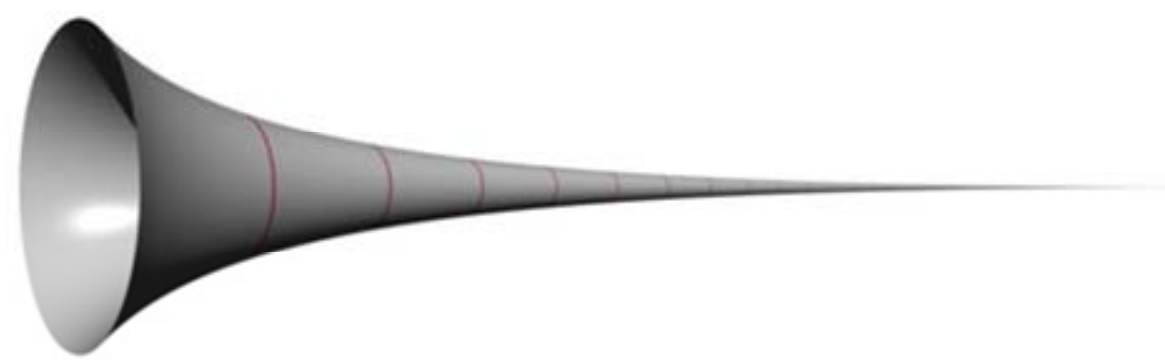

Figure 2.

Torricelli's trumpet (Image from http://en.wikipedia.org/wiki/File:GabrielHorn.png).

However, the horn evidently exists in the abstract, mathematical realm where the infinitely large and infinitely small readily co-exist. Without finite molecules to worry about, we can see that the horn's internal volume is also infinitely long as it extends into the endless and infinitely narrow tube. Obviously, a volume with an infinite dimension is definitely not finite. This presents no difficulty because $\pi$ is not a finite integer. It is a transcendental number and has an infinite decimal expansion with cardinality $\aleph_{0}$.Using Cantor's results, we can see that there is a one-to-one correspondence between the points in the horn's volume and the points on its surface. In fact, we already mentioned earlier how the numbers of points on any surface and in any volume are infinite, with the same cardinality $\mathbf{c}$.

This may seem counter-intuitive, but it is in keeping with the even integers having the same cardinality $\aleph_{0}$ as all integers (odd and even) together, and with the real numbers in the interval $(0,1)$ having the same cardinality $\mathbf{c}$ as the real numbers in any other interval (smaller or larger). A comparison can be made with the points on the surface of an abstract pea having a one-to-one correspondence with the points on the surface of an abstract star, both with cardinality c. This does not apply to concrete stars and concrete peas but it is mathematically sound. Let us note here that a drop of abstract paint is all we need to paint an infinite object. It suffices to apply an infinitely thin layer of paint (i.e. a trick we cannot do with concrete molecules of paint).

The horn's paradox thus disappears. It only exists when we mistakenly attempt to apply the properties of abstract infinity to finite matter. In abstractness, where there is no physical distance, infinity takes no room; there is no physical difference between infinitely large and infinitely small abstractions. Perhaps the most interesting aspect of an abstract Gabriel's horn is that when one uses infinitely small units to build it, the horn is both infinitely small and infinitely long.

\section{Infinity and Quantum Physics}

The rules of quantum physics are also counter-intuitive and paradoxical in their own way. They radically differ from those of classical physics and include the apparent possibility of being at two places at once, the wave-particle duality problem, the quantum entanglement of sub-atomic particles, the popping up of virtual particles from the vacuum, quantum tunnelling, and the fundamental importance of infinite probabilities and complex numbers (with non-zero imaginary parts). This is very different from what occurs in the case of concrete matter. None of these phenomena make sense in classical physics, but all are crucial and fundamental in quantum mechanics. The wave- particle riddle has particularly struck the popular imagination because it immediately caused disagreement and controversy amongst the leading physicists of the time when it was first discovered. Eight decades later, physics textbooks, scientific journals and popular science magazines still present this duality as weird and defying common sense although scientifically proved beyond doubt: depending on the type of measuring apparatus one choses, photons and other quantum elements turn out to behave either like waves or like particles, and their strange, fundamental nature remains elusive.

For the purpose of this article, the importance of infinity and imaginary numbers is especially relevant. In classical physics, infinities are always truncated, and imaginary numbers are simply used as mathematical tricks to solve complex problems without altering our view of the world. In quantum physics, on the contrary, infinities and imaginary values are inescapable and absolutely essential for comprehension and ontology.

It is important at this point to consider the following argument. When we throw a single die, we expect one of six results. If presented with a new game that produces twelve possible results, we know that two throws (or two dice) must be involved. If we randomly pick up numbered balls from a bag and only ever get numbers from 1 to 100 , we can reasonably conclude that the bag must contain a minimum of 100 balls numbered from 1 to 100. Similarly, the fundamental importance of infinite probabilities in quantum physics implies the background presence of an infinite and therefore abstract source. This means that the existence of quantum particles depends on the existence of abstract infinity.

If one accepts this, one must logically conclude that we live in a Universe where quantum particles lie at the interface between the abstract and concrete aspects of reality.

\section{Mathematical Platonism}

Infinity is an abstract concept not concretised in physical matter, but it does exist. All abstract ideas do exist even if we cannot touch them. In fact, existence out of space-time is confidently assumed by those mathematicians who believe in mathematical Platonism, the idea that mathematical statements literally exist in an abstract realm independent of rational observers.

It is standard practice to accept at least a watered-down version of mathematical Platonism restricted to the first two items of the definition: 1) infinity exists and 2) it is abstract. Whether it exists independently of rational observers is still an object of mathematical and philosophical debate, but if infinite probabilities have governed the production and existence of quantum particles since the Big Bang, their presence at the beginning of 
the concrete Universe definitely did not depend on rational observers born more than 13 billion years later. Viewed in this light, the full version of mathematical Platonism becomes a logical necessity and an essential prerequisite for our very existence.

Believing in the consequential reality of abstractness is a mental process comparable to the widespread belief in a Heaven that exists out of space-time or in a Nirvana where one can escape from space-time. Philosophically, the full version of mathematical Platonism means that mathematicians do not invent theorems, but discover them. In quantum physics, it gives an altogether new significance to the equations that precisely describe quantum elements, by making us realise that quanta are neither waves nor particles but are mathematically virtual, intangible entities. Photons, for instance, are well known to be discrete packets of pure energy without concrete substance. Ever since they were discovered, they have been fully described as mathematical functions, i.e. intangible entities. It is only when they are detected that they lose their virtual character and integrate concrete space-time.

For mathematical Platonists who consider the relationship between infinity and quantum mechanics, it is natural to accept the view that the quantum particles making up the universe first pop up into the vacuum out of an abstract and infinite source. Such a mathematical interpretation is indeed much more logical (or at least more palatable) than the currently popular view among physicists that our universe sprang out of "nothing" (Hawking \& Mlodinow, 2010; Maxwell, 2011; Krauss, 2012; Holt, 2012; Set \& Rêve, 2012; Côté, 2012). An origin from abstractness also has some commonality with religious creation dogmas developed throughout human history by people who knew little about the properties of infinity and, for lack of a better explanation, resorted to imagining various anthropomorphic gods. In fact, an origin from abstractness is supported by the elegant equation first discovered by the Swiss mathematician and physicist Leonhard Euler (1707-1783) who showed that the imaginary power of an imaginary number can be a real number:

$$
i^{i}=1 / \sqrt{ }\left(e^{\pi}\right)
$$

In summary, we have now established the following logical sequence: the sheer existence of abstract infinity implies endless self-reference, the existence of further power sets, greater infinity, the production of virtual quantum particles and, from the known combinations of these particles, the consequent formation of space-time. In other words, the existence of abstract infinity is the basic reason why, in the concrete universe, there is something rather than nothing.

\section{Conclusion}

The solutions proposed above, often counter-intuitive, are far reaching and can hopefully encourage further research and discussion until mathematicians and philosophers make sense of this most logical topic and quench our inborn thirst for the absolute and the Infinite.

\section{Acknowledgements}

The constructive criticism and encouragement of Prof. Lucien Pelletier of the Dept. of Philosophy, University of Sudbury (Ontario) is gratefully acknowledged.

\section{REFERENCES}

Clegg, B. (2003). A brief history of infinity. The quest to think the unthinkable. London: Robinson.

Cole, J. C. (2010). Mathematical platonism. Internet Encyclopedia of Philosophy. http://www.iep.utm.edu/mathplat/

Côté, G. B. (2012). Latest quantum experiments point to the reality of abstractness and timelessness. http://comments.sciencemag.org/content/10.1126/science. 1226755

Dunham, W. (1990). Journey through genius. The great theorems of mathematics. New York: Penguin Books, John Wiley \& Sons.

Dunham, W. (1994). The mathematical universe. An alphabetical journey through the great proofs, problems, and personalities. New York: John Wiley \& Sons.

Hawking, S., \& Mlodinow, L. (2010). The grand design. New York: Bantam Books, Random House.

Holt, J. (2012). Why does the world exist? An existential detective story. New York: Liveright Publishing, W. W. Norton.

Krauss, L. M. (2012). A universe from nothing. Why there is something rather than nothing. New York: Free Press.

Maxwell, N. (2011). Three philosophical problems about consciousness and their possible resolution. Open Journal of Philosophy, 1, 1-10. doi:10.4236/ojpp.2011.11001

Set, J., \& Rêve, J. (2012). Our theory of everything. Raleigh, NC: Lulu.

Weisstein, E. W. (2013). Gabriel's horn. Math World-A Wolfram Web Resource. http://mathworld.wolfram.com/GabrielsHorn.html

Wikipedia, Gabriel's horn. http://en.wikipedia.org/wiki/Gabriel's Horn 